\title{
Central stars of planetary nebulae: New spectral classifications and catalogue ${ }^{\star}$
}

\author{
W. A. Weidmann ${ }^{1, \star \star}$ and R. Gamen ${ }^{2, \star \star \star}$ \\ ${ }^{1}$ Observatorio Astronómico Córdoba, Universidad Nacional de Córdoba, Argentina \\ e-mail: walter@mail .oac . uncor . edu \\ 2 Instituto de Astrofísica de La Plata, CCT La Plata-CONICET, Universidad Nacional de La Plata, Argentina \\ e-mail: rgamen@fcaglp.unlp.edu.ar
}

Received 29 December 2009 / Accepted 24 October 2010

\begin{abstract}
Context. There are more than 3000 confirmed and probable known Galactic planetary nebulae (PNe), but central star spectroscopic information is available for only $13 \%$ of them.

Aims. We undertook a spectroscopic survey of central stars of PNe at low resolution and compiled a large list of central stars for which information was dispersed in the literature.

Methods. We observed 45 PNs using the $2.15 \mathrm{~m}$ telescope at Casleo, Argentina.

Results. We present a catalogue of 492 confirmed and probable CSPN and provide a preliminary spectral classification for 45 central star of PNe. This revises previous values of the proportion of CSPN with atmospheres poor in hydrogen in at least 30\% of cases and provide statistical information that allows us to infer the origin of $\mathrm{H}$-poor stars.
\end{abstract}

Key words. surveys - planetary nebulae: general - stars: evolution

\section{Introduction}

A planetary nebula is the most luminous transitory phase in the life of low and intermediate mass stars $\left(0.6 M_{\odot}<M<8 M_{\odot}\right)$ on their evolution from the asymptotic giant branch (AGB) to their final destiny, white dwarfs (WD). The PN phase begins once the central star reaches an effective temperature of $30000 \mathrm{~K}$ and ionises the shell of material ejected during its evolution in the AGB. After about about $2 \times 10^{4}$ years, it ends when the nuclear burning in a thin shell of the star stops, and the nebula finally disperses.

PNe were discovered more than two centuries ago, and their number has increased every year, but there are still unsolved questions about them. Some of these, and perhaps the most important ones, are related to aspects of the central stars of the planetary nebulae (CSPN). Planetary nebulae nuclei are not located in a confined region of the HR diagram, and their optical spectra encompass all varieties known for hot stars, i.e. ranging from pure emission to emission-absorption mixtures and from near-continuous to pure strong absorption. The appearance of the spectrum depends upon temperature, luminosity, and chemical composition, or more fundamentally, upon core mass and state of evolution. Méndez (1991) suggested that the majority of CSPN can be classified in two distinct categories: those for which stellar $\mathrm{H}$ features can be identified in

^ Based on data collected at the Complejo Astronómico El Leoncito (CASLEO), which is operated under agreement between the Consejo Nacional de Investigaciones Científicas y Técnicas de la República Argentina y Universidades Nacionales de La Plata, Córdoba y San Juan, Argentina.

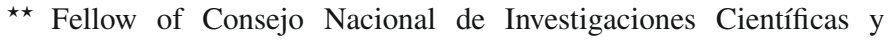
Técnicas (CONICET), Argentina.

$\star \star \star$ Member of Carrera del Investigador CONICET, Argentina. their spectra (hydrogen-rich) and those for which they cannot (hydrogen-poor).

At present, there are about 3000 confirmed and probable $\mathrm{PNe}$ known in our Milky Way, listed in Acker et al. (1992, 1996) $\left(\right.$ SECGPN $\left.^{1}\right)$, (Parker et al. 2006; and Miszalski et al. 2002) $\left(\mathrm{MASH}^{2}\right.$ ), and Drew et al. (2005) (IPHAS, INT Photometric H-Alpha Survey). However, spectroscopic information on their central stars is known only in a very small fraction of objects (about 13\%, see Sect. 3).

Spectroscopy of CSPN is difficult to obtain because of their apparent low brightness, low apparent magnitudes (60\% of the CSPN listed in the SECGPN have $V>15.5$ ), and the surrounding gaseous shell whose emission lines often mask the stellar lines. In addition, the position of the CSPN is not always clear.

The determination of spectral types of CSPN should help significantly to improve our knowlege of their general evolutionary scheme, making it possible to consider CSPN as physical objects with individual parameters and peculiarities and not just as sources of ionizing radiation.

One of the first lists of CSPN was compiled by Aller (1948), then another was produced by Acker et al. (1982) (Catalogue of CSPN, Strasbourg Observatory). Information on CSPN can be found in the SECGPN and the MASH CDS-catalogues. Several authors have added contributions, although often for particular spectral types, e.g. WR+wels (Acker \& Neiner 2003), B[e] (Lamers et al. 1998), evolved CSPN (Napiwotzki 1999), and PG 1159 (Werner \& Herwig 2006).

\footnotetext{
${ }^{1}$ Strasbourg-ESO Catalogue of Galactic PN (SECGPN) http:// vizier.u-strasbg.fr/viz-bin/VizieRPlanetary_NebulaeV/ $84 /$ cstar.

${ }^{2}$ Macquarie/AAO/Strasbourg $\mathrm{H} \alpha$ Planetary Galactic Catalog http://vizier.u-strasbg.fr/vizier/MASH
} 
Table 1. Summary of the spectral types of CSPN compiled in our catalogue, grouped by their atmospheric hydrogen abundance.

\begin{tabular}{|c|c|c|c|c|c|}
\hline \multicolumn{3}{|c|}{ H-rich } & \multicolumn{3}{|c|}{ H-poor } \\
\hline Sp.Type & Sample & Sp. Type & Sample & Sp. type & Sample \\
\hline O3-9+B early & 64 & $\mathrm{sdB}$ & 1 & [WC4-11] & 57 \\
\hline Of & 20 & Hybrid & 3 & [WO1-4] & 33 \\
\hline Later that B5 & 38 & Symbiotic star? & 7 & [WR] & 11 \\
\hline $\mathrm{B}[\mathrm{e}]$ & 6 & Blue & 50 & [WN] & 5 \\
\hline $\mathrm{DA}+\mathrm{WD}$ & 12 & Emission-line & 25 & PG 1159 & 15 \\
\hline $\mathrm{DAO}$ & 14 & & & [WC]-PG1159 & 2 \\
\hline $\mathrm{sdO}$ & 3 & & & $\mathrm{O}(\mathrm{He})$ & 3 \\
\hline $\operatorname{hgO}(\mathrm{H})$ & 16 & & & $\mathrm{O}(\mathrm{c})+\mathrm{Of}(\mathrm{c})$ & 2 \\
\hline Cont. & 16 & & & H-poor & 1 \\
\hline H-rich & 3 & & & DO & 4 \\
\hline & & & & wels & 72 \\
\hline Total & 192 & Total & 86 & Total & 205 \\
\hline
\end{tabular}

Notes. Here, we have discarded 9 objects without any specific spectral type.

To contribute to the knowledge of the final stellar evolution stages, we undertook a spectroscopic survey of CSPN and compiled a large list of CSPN. The motivation of the present work lies in a series of astronomical concerns: the complicated puzzle of different types of CSPN observed (see Table 1), few stars with spectral information, a lack of consensus in the evolutionary sequence of the CSPN, and the surprising bimodality in their hydrogen abundance.

This paper is organized as follows. The sample and observations are described in Sect. 2.1; in Sect. 2.2, we comment on the spectral classification; in Sect. 3, we present the catalogue of CSPN and we give a brief discussion. Finally, in Sect. 4 we present our conclusions.

\section{New spectral classification}

\subsection{Observations}

We observed 45 southern CSPN selected from SECGPN and Boumis et al. (2003), the coordinates of which were taken from Kerber et al. (2003).

The observations were carried out during a three-year campaign between 2005 November and 2008 December that included a total of 31 nights of observations. For this survey, we used the REOSC spectrograph attached to the 2.15-m telescope at CASLEO, Argentina.

A 300 line $\mathrm{mm}^{-1}$ grating was used, which yielded a dispersion of $3.4 \AA$ pixel $^{-1}$. During some nights, a grating of 600 line $\mathrm{mm}^{-1}$ was used $\left(1.6 \AA\right.$ pixel $\left.^{-1}\right)$. The gratings provide a typical wavelength range of 3500-7000 $\AA$ (3875-5530 $\AA$ for the highest resolution). The slit was opened to $3^{\prime \prime}$ to be consistent with the seeing at the site.

\subsection{Results}

In this first work, we present a very preliminary classification of the observed CSPN. We distinguish between CSPN with absorption and emission lines. In the former group, we basically identified absorption lines of He I and He II, these CSPN then being classified as OB. The latter group contained CSPN with identified emission lines, mainly of CIII (4650 ̊ and $5696 \AA$ ) and C IV (5806 $\AA$ ), which are typical of [WC] stars. This CSPN were classified as "emission-line". We obtained some spectra
Table 2. Summary of results of KS test applied to the sample of Galactic latitude.

\begin{tabular}{lcr}
\hline \hline $\begin{array}{l}\text { Compared } \\
\text { groups }\end{array}$ & $D$ & \multicolumn{1}{c}{$P$} \\
\hline H-rich vs. H-poor & 0.26 & $<0.1 \%$ \\
H-rich vs. wels & 0.25 & $0.3 \%$ \\
H-poor vs. wels & 0.11 & $64.1 \%$ \\
\hline
\end{tabular}

Notes. Where $D$ indicates the differences between the cumulative number distributions and $P$ the probability that the compared samples are equal.

whose stellar continuum had a reasonable signal-to-noise ratio $(\mathrm{S} / \mathrm{N})$, but displayed, neither absorption nor emission lines. In these cases, although classified as "continuous" type, these objects are axpected to be H-rich (Kudritzki et al. 1981). Result are shown in Table 3. In a forthcoming paper, we perform a detailed spectroscopic analysis.

\section{The catalogue of CSPN}

\subsection{Content}

Taking into account that the information about CSPN spectral types is scattered among many publications, we carried out an extensive bibliographic compilation of the CSPN data with the goal of producing an updated list of those stars that have spectroscopic information. This list includes 492 stars of both confirmed and possible PN with spectral-type determinations, 45 of them from our own new data. Transition objects, such as post-AGB, PPN, or young-PN (Ej. V 348 Sgr, CRL 618, He 1-5, BD+33 2642, LS IV-12 111 and He 3-1475) were not included.

The information included in the catalogue, discriminated between being confirmed and possible PN (Table 4), is:

Col. 1 the PN G designation, taken from SECGPN;

Col. 2 the common name of the object;

Cols. 3-4 the equatorial coordinates (J2000.0) of the nebula, since in most cases there is no information on the position of the CSPN. Though in many cases this is evident, in others it is not;

Col. 5 the spectral classification of the CSPN. If there are more than one, they are separated by a semi-colon (idem in the references column). However, we use only two spectral classifications if is it necessary, for example when the spectral classifications are very different. When the authors observed Balmer series absorption, we labeled these objects as H-rich. In some cases, the authors do not give the spectral type of the $\mathrm{CS}$, but describe the identified lines. We also include the CSPN classified by Miszalski et al. (2002) in the MASHII catalogue: blue, [WR] or wels. Note that the blue characteristics of the CSPN images in MASHII is not based on any spectroscopic study;

Col. 6 the reference where the spectral type was found (t.w. means this work);

Col. 7 the reference that indicates whether the star is part of a binary system (nothing if not). Although some CSPN are of a late MK spectral type, it is accepted that the excitation source of the PN (if star and nebulae are physically associated) is a hitherto undetected hot star (Lutz 1977). In those cases, we include the label bc-CSPN, corresponding to binarity for the cool CSPN. 

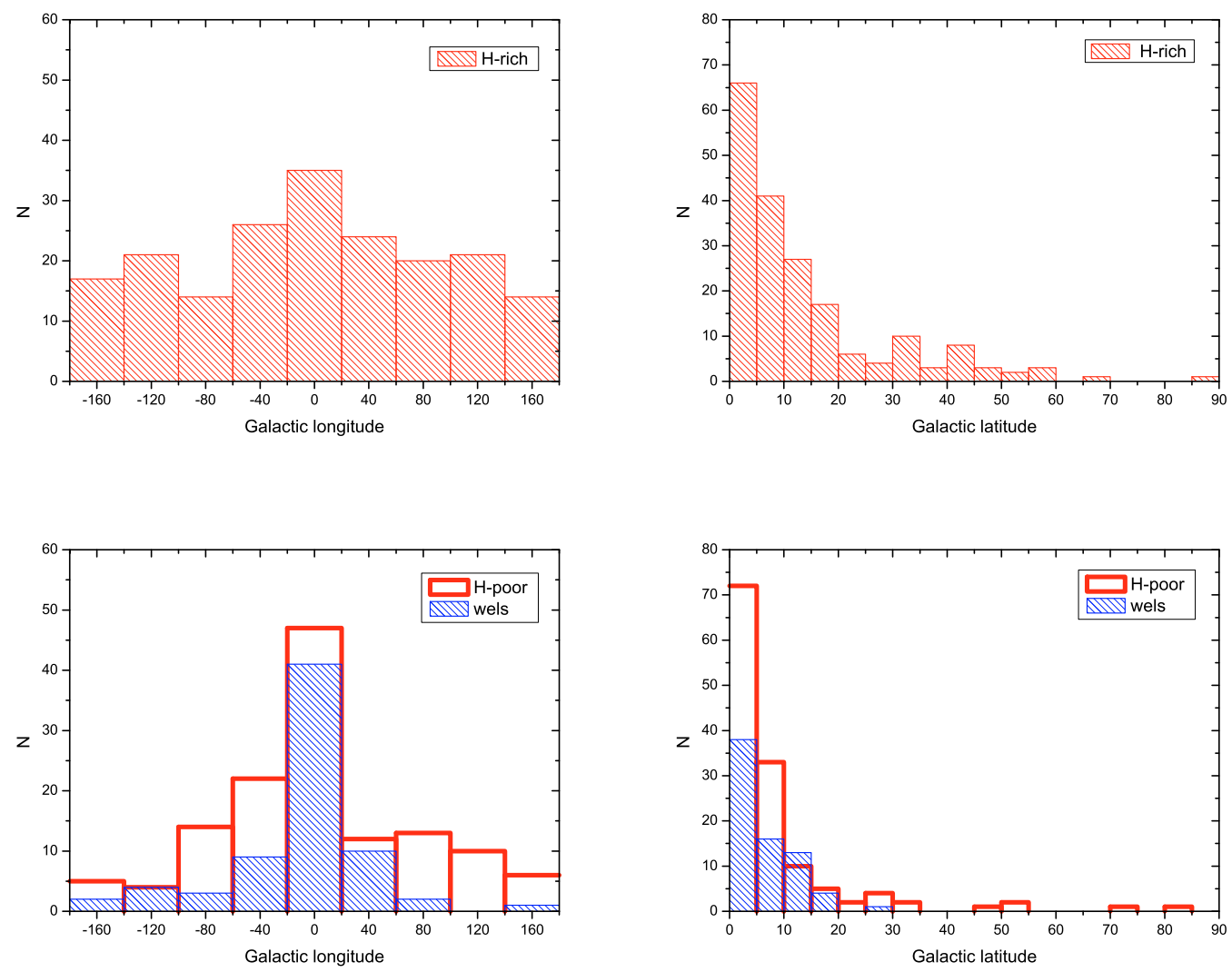

Fig. 1. Distributions in Galactic longitude and latitude of CSPN (of true and possible PN) that belong to H-rich, H-poor, and wels star groups. Note that H-poor PN are more concentrated towards the Galactic center than H-rich ones. The similarity between the wels and H-poor distributions is also noticeable.

The catalogue of Acker et al. (1992) and AN03 provided spectroscopic information for 240 CSPN; with this new collated list, the number of CSPN with spectral classification has doubled. We hope that this new list will be useful for future investigations. In addition, we note that Parker et al. (2006) estimated that $\sim 30 \%$ of the MASH entries have candidate CSPN, with about half of these being high quality candidates suitable for immediate follow-up, so the list of CSPN with spectral classification will be increased quickly.

\subsection{Discussion}

The larger sample of CSPN with spectral types allows us to discuss the dichotomy between $\mathrm{H}$ rich and poor stars.

We grouped the H-rich and H-poor CSPN in Table $1^{3}$, It is clear that the former group is more numerous than the H-poor one, the ratio being 1.4. In an earlier study, Méndez (1991) reported a ratio of 3 . It is evident that stars with strong emission lines are easier to detect than those with absorption lines, thus favouring the detection of $\mathrm{H}$-poor stars. However, is this effect strong enough to explain the ratio of stars observed between both groups?

We have found above that $30 \%$ of the whole CSPNe population appears to be hydrogen deficient (without counting the

\footnotetext{
3 Although we have included the wels in the H-poor group (since we found evidence that wels and H-poor are in the same group), we prefer to be cautious and define and use the three groups $\mathrm{H}$-rich, $\mathrm{H}$-poor, and wels in the following discussion.
}

"blue" stars). It is difficult to obtain a theoretical prediction of this ratio of stellar types because the mechanism for generating H-poor CSPNe is not well known. The more accepted hypothesis for explaining the lack of hydrogen in the atmospheres of CSPN is the born-again phenomenon (Iben et al. 1983). In this framework, it is estimated that roughly $15 \%$ (Lawlor \& MacDonald 2001) of post-AGB stars suffer a born-again event. Blöcker et al. (2001), based on their improved born-again models (thermal pulses plus overshooting), found that $20-25 \%$ of stars can be expected to become H-poor. These theoretical values are substantially lower than our observational value. According to this catalogue, it is difficult to imagine how a selection effect could be as efficient as to produce this high fraction of H-poor stars, so perhaps the born-again phenomenon is not the unique mechanism for obtaining an atmosphere free of hydrogen. We recall other ways to form H-poor CSPN, such as the binary channel (Tylenda \& Górny 1993) or the continuous stripping of the outer H-rich layers by intense stellar winds (Górny \& Tylenda 2000).

Only 71 close binary CSPN have been found (de Marco 2009; Miszalski et al. 2009b, and 2010), almost all of which have a H-rich spectra. The first [WR] star, in a close binary system, has been discovered in 2010 (Hajduk et al. 2010). We note that nearly $14 \%$ of the compiled CSPN are probably binary systems, in good agreement with the 10-15 value obtained by Bond et al. (1989).

We analyzed the distribution in Galactic coordinates of the CSPN sample that belongs to the H-rich and H-poor groups. 
Table 3. Spectral types from our observations.

\begin{tabular}{|c|c|c|c|c|c|}
\hline Name & PN G & $\mathrm{AR}(2000)$ & $\operatorname{Dec}(2000)$ & Sp. Type & E.T.[s] (grating) \\
\hline H $1-62$ & $000.0-06.8$ & 181317.9 & -321943.0 & emission-line & $3600(300)$ \\
\hline PC 12 & $000.1+17.2$ & 164349.3 & -185633.0 & $\mathrm{OB}$ & $2 \times 1200(300)$ \\
\hline IC 4634 & $000.3+12.2$ & 170133.5 & -214933.1 & emission-line & $3 \times 1000(300)$ \\
\hline H 1-63 & $002.2-06.3$ & 181618.5 & -300735.8 & $\mathrm{OB} ?$ & $3600(300)$ \\
\hline M 1-38 & $002.4-03.7$ & 180605.8 & -284034.3 & cont. & $3600(300)$ \\
\hline M 1-53 & $015.4-04.5$ & 183548.2 & -173608.4 & emission-line? & $3600(300)$ \\
\hline Sa $1-8$ & $020.7-05.9$ & 185044.2 & -133102.4 & OB & $3600(300)$ \\
\hline IRAS $19021+0209$ & 036.4-01.9 & 190438.5 & 021423.0 & cont. & $3600(300)$ \\
\hline M 1-6 & $211.2-03.5$ & 063544.6 & -000541.1 & emission-line & $3600(300)$ \\
\hline SaSt 2-3 & $232.0+05.7$ & 074803.5 & -140742.6 & $\mathrm{OB}$ & $3600(300)$ \\
\hline M 1-11 & $232.8-04.7$ & 071116.6 & -195103.0 & emission-line & $3600(300)$ \\
\hline M 1-14 & 234.9-01.4 & 072756.5 & -20 1323.4 & $\mathrm{OB}$ & $2 \times 3600(300)$ \\
\hline M 1-12 & $235.3-03.9$ & 071921.4 & -214355.3 & emission-line & $3600(300)$ \\
\hline Y-C 2-5 & $240.3+07.0$ & 081041.7 & -203132.9 & emission-line & $3600(300)$ \\
\hline KLSS 1-9 & $240.8-19.6$ & 062436.4 & -330449.0 & $\mathrm{OB}$ & $3600(300)$ \\
\hline M 3-4 & $241.0+02.3$ & 075511.2 & -233745.6 & cont. & $3600(300)$ \\
\hline M 3-1 & $242.6-11.6$ & 070249.6 & -313541.3 & cont. & $3600(300)$ \\
\hline M 4-2 & $248.8-08.5$ & 072855.2 & -354515.4 & emission-line & $3600(300)$ \\
\hline Ns 238 & $254.6+00.2$ & 082056.7 & -361346.7 & $\mathrm{OB}$ & $2 \times 3600(300)$ \\
\hline PB 2 & $263.0-05.5$ & 082039.8 & -462013.2 & emission-line? & $2 \times 1200(300)$ \\
\hline PB 4 & $275.0-04.1$ & 091507.6 & -545238.5 & emission-line? & $3600(300)$ \\
\hline IC 2501 & $281.0-05.6$ & 093847.5 & -600527.9 & emission-line & $2 \times 3600(300)$ \\
\hline IC 2553 & $285.4-05.3$ & 100921.7 & -623640.9 & emission-line & $4 \times 300(300)$ \\
\hline He 2-47 & $285.6-02.7$ & 102309.0 & -603234.3 & emission-line & $2 \times 2700(300)$ \\
\hline IC 2621 & $291.6-04.8$ & 110019.5 & -651454.2 & emission-line & $2 \times 3600(300)$ \\
\hline Lo 6 & $294.1+14.4$ & 120043.5 & -473312.0 & cont. & $3600(300)$ \\
\hline Th 2-A & 306.4-00.6 & 132234.8 & -63 2055.2 & emission-line & $3600(300)^{a}$ \\
\hline He 2-97 & $307.2-09.0$ & 134524.0 & -712848.8 & emission-line & $3600(300)$ \\
\hline He 2-105 & $308.6-12.2$ & 141525.7 & -741249.8 & OB & $3600(300)$ \\
\hline NGC 5307 & $312.3+10.5$ & 135103.3 & -511215.9 & emission-line & $3600(300)$ \\
\hline He 2-107 & $312.6-01.8$ & 141842.5 & -630710.7 & emission-line & $2 \times 3600(300)$ \\
\hline He 2-434 & $320.3-28.8$ & 193350.7 & -743258.7 & OB & $3600(300)$ \\
\hline NGC 5979 & $322.5-05.2$ & 154740.6 & $\begin{array}{llll}-61 & 13 & 02.7\end{array}$ & emission-line & $2 \times 1500(300)$ \\
\hline He $2-128$ & $325.8+04.5$ & 152507.9 & -511940.9 & emission-line? & $3600(600)$ \\
\hline WRAY 17-75 & $329.5-02.2$ & 161234.4 & -542335.3 & OB & $3600(300)$ \\
\hline He 2-187 & $337.5-05.1$ & 170137.4 & -502256.6 & OB & $3600(300)$ \\
\hline NGC 6026 & $341.6+13.7$ & 160120.8 & -343238.0 & $\mathrm{OB}$ & $3600(300)$ \\
\hline PC 17 & $343.5-07.8$ & 173541.1 & -465951.3 & emission-line & $3600(600)$ \\
\hline Cn 1-3 & $345.0-04.9$ & 172611.8 & -441129.1 & emission-line? & $4 \times 700(300)$ \\
\hline IC 4663 & $346.2-08.2$ & 174528.5 & -445411.5 & emission-line? & $4 \times 700(600)$ \\
\hline IC 4699 & $348.0-13.8$ & 181831.2 & -455903.2 & emission-line & $3600(600)$ \\
\hline NGC 6337 & $349.3-01.1$ & 172216.0 & -382857.6 & emission line & $3600(300)$ \\
\hline Fg 3 & $352.9-07.5$ & 180011.9 & -384951.7 & cont. & $3600(300)$ \\
\hline H 1-35 & $355.7-03.5$ & 174913.9 & -342253.3 & emission-line? & $2 \times 1700(300)$ \\
\hline Te 2022 & $358.8-00.0$ & 174242.4 & -295135.4 & OB & $3600(300)$ \\
\hline
\end{tabular}

Notes. The PNe are denoted by their common name and by their PN G designation. Fifth column lists the preliminary spectral type that we have

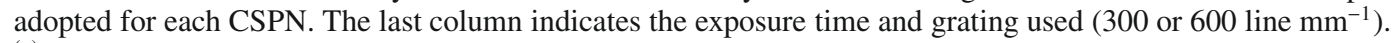

(a) GEMINI observation, see Weidmann et al. (2008).

From Fig. 1, it is evident that there is a strong concentration of H-poor and wels stars toward the Galactic center. This effect was observed by Górny et al. (2004) and attributed to a possible selection effect. However, it might be caused by the influence of metallicity in the mechanism that leads to an unleashing of the total hydrogen loss from the stellar atmosphere of those objects.

On the other hand, the average height above the Galactic plane of H-rich, H-poor and wels stars was found to be $13.9^{\circ} \pm$ $15.2,9.0^{\circ} \pm 12.6$, and $6.7^{\circ} \pm 5.3$, respectively. As these errors are too large, we performed a Kolmogorov-Smirnov (KS) statistical analysis. The significance of the trends in KS test is assessed on the basis of differences, $D$, between their cumulative number distributions. This is used to define a probability coefficient $P$, such that low values of $P$ imply significant differences. The results of the KS test are shown in Table 2. It is clear that the distribution of Galactic latitudes of $\mathrm{H}$-rich and $\mathrm{H}$-poor stars are very different. In addition, the sample of wels stars are, apparently, more similar to the H-poor stars than the other group, supporting the hypothesis that wels stars belong to the H-poor group and enhancing the ratio of $\mathrm{H}$-poor to the whole CSPN population.

\section{Conclusions}

We have carried out a spectroscopic survey of PNe, during which we have performed a very preliminary determination of the spectral types of 45 of their central stars, all of them previously unclassified. In addition, we have performed an extensive bibliographic compilation of CSPN with determined spectral 
types. We have presented the list of 492 CSPN with spectral classification (together with their respective references), and included a tag indicating those that are either binary systems or candidates. We hope that this list will be useful for future investigations.

From our catalogue, we grouped CSPN whose atmospheres are hydrogen rich or poor; conservatively we ruled out the wels (nevertheless we found evidence supporting the hypothesis that wels belong to the H-poor group). We found that the ratio of stars in both groups is lower than previous estimates. According to our statistical analysis, we have found that PN with H-poor central star are more concentrated toward the Galactic center and Galactic plane than the $\mathrm{H}$-rich group. This suggests that $\mathrm{H}-$ poor stars may have a more massive progenitor and in addition, the metallicity could play an important role in the mechanism responsible for generating hydrogen-free atmospheres. In addition, we have found that the frequency of occurrence of known close binaries among CSPNe is $\sim 14 \%$.

Acknowledgements. We would like to thank our anonymous referee whose critical remarks helped us to substantially improve this paper. The CCD and data acquisition system at CASLEO has been financed by R. M. Rich trough US NSF grant AST-90-15827. This work has been partially supported by Concejo de Investigaciones Cientifícas y Técnicas de la República Argentina (CONICET). This research has made use of the SIMBAD database, operated at CDS, Strasbourg, France. The authors wish to thank Drs. Guillermo Bosch and Roberto H. Méndez for comments that helped to improve the paper.

\section{References}

Abell, G. O. 1966, ApJ, 144, 259

Acker, A., \& Neiner, C. 2003, A\&A, 403, 659

Acker, A., Gleizes, F., Chopinet, M., et al. 1982, CDSSP, 3

Acker, A., Marcout, J., Ochsenbein, F., Stenholm, B., \& Tylenda, R. 1992,

Strasbourg-ESO catalogue of galactic planetary nebulae (Garching: European Southern Observatory)

Aller, L. H. 1948, ApJ, 108, 462

Aller, L. H., \& Keyes, C. D. 1985, PASP, 97, 1142

Aller, L. H., \& Keyes, C. D. 1987, ApJS, 65, 405

Aller, L. H., Hyung, S., \& Feibelman, W. A. 1996, PASP, 108, 488

Belczyński, K., Mikolajewska, J., Munari, U., Ivison, R. J., \& Friedjung, M. 2000, A\&AS, 146, 407

Benetti, S., Cappellaro, E., Ragazzoni, R., Sabbadin, F., \& Turatto, M. 2003, A\&A, 400, 161

Bianchi, L., \& Defrancesco, G. 1993, IAUS, 155, 85

Bilikova, J., Chu, Y.-H., Su, K., et al. 2008, in 16th European White Dwarf Workshop, ASP Conf. Ser., in press

Blöcker, T., Osterbart, R., Weigelt, G., Balega, Y., \& Men'shchikov, A. 2001, ASSL, 265, 241

Bohigas, J. 2008, ApJ, 674, 954

Bond, H. E., \& Ciardullo, R. 1999, PASP, 111, 217

Bond, H. E., \& Grauer, A. D. 1987, Second Conference on Faint Blue Stars, ed.

A. G. D. Philip, D. S. Hayes, \& J. W. Liebert, IAU Colloq., 95, 221

Bond, H. E., \& Livio, M. 1990, ApJ, 355, 568

Bond, H. E., \& Pollacco, D. L. 2002, AP\&SS, 279, 31

Bond, H. E., Ciardullo, R., \& Meakes, M. 1989a, BAAS, 21, 789

Bond, H. E., Ciardullo, R., Fleming, T. A., \& Grauer, A. D. 1989b, IAUS, 131, 310

Bond, H. E., Meakes, M. G., Liebert, J. W., \& Renzini, A. 1993, IAUS, 155, 499

Bond, H. E., O’Brien, M. S., Sion, E. M., et al. 2002, ASPC, 279, 239

Bond, H. E., Pollacco, D. L., \& Webbink, R. F. 2003, AJ, 125, 260

Boumis, P., Paleologou, E. V., Mavromatakis, F., \& Papamastorakis, J. 2003, MNRAS, 339, 735

Brocklehurst, M. 1971, MNRAS, 153, 471

Cerruti-Sola, M., \& Perinotto, M. 1985, ApJ, 291, 237

Chromey, F. R. 1980, AJ, 85, 853

Chu, Y., Gruendl, R. A., Guerrero, M. A., et al. 2009, AJ, 138, 691

Ciardullo, R., Bond, H. E., Sipior, M. S., et al. 1999, AJ, 118, 488

Cohen, M., \& Jones, B. F. 1987, ApJ, 321, L151

Corradi, R. L. M. 1995, MNRAS, 276, 521

de Marco, O. 2006, IAUS, 234, 111

de Marco, O. 2009, PASP, 121, 316

de Marco, O., Sandquist, E. L., Mac Low, M. M., Herwig, F., \& Taam, R. E. 2003, RMxAC, 18, 84
Dreizler, S. 1999, RvMA, 12, 255

Drew, J. E., Greimel, R., Irwin, M. J., et al. 2005, MNRAS, 362, 753

Drilling, J. S. 1983, ApJ, 270, L13

Drilling, J. S. 1985, ApJ, 294, L107

Duerbeck, H. W., \& Benetti, S. 1996, ApJ, 468, L111

Exter, K. M., Pollacco, D. L., Maxted, P. F. L., Napiwotzki, R., \& Bell, S. A. 2005, MNRAS, 359, 315

Feibelman, W. A. 1994, PASP, 106, 56

Feibelman, W. A. 1999, PASP, 111, 719

Feibelman, W. A., \& Kaler, J. B. 1983, ApJ, 269, 592

Ferguson, D. H., McGraw, J. T., Spinrad, H., Liebert, J., \& Green, R. F. 1981, ApJ, 251, 205

Frew, D. J., Parker, Q. A., \& Russeil, D. 2006, MNRAS, 372, 1081

Frew, D. J., Stanger, J., Fitzgerald, M., et al. 2010, PASA, in press

Gauba, G., Parthasarathy, M., Nakada, Y., \& Fujii, T. 2001, A\&A, 373, 572

Gesicki, K., \& Zijlstra, A. A. 2003, MNRAS, 338, 347

Gesicki, K., Zijlstra, A. A., Acker, A., et al. 2006, A\&A, 451, 925

Górny, S. K., \& Siódmiak, N. 2003, IAUS, 209, 43

Górny, S. K., \& Tylenda, R. 2000, A\&A, 362, 1008

Górny, S. K., Stasinska, G., Escudero, A. V., \& Costa, R. D. D. 2004, A\&A, 427, 231

Górny, S. K., Chiappini, C., Stasinska, G., \& Cuisinier, F. 2009, A\&A, 500, 1089 Grauer, A. D., \& Bond, H. E. 1983, ApJ, 271, 259

Grauer, A. D., Bond, H. E., Ciardullo, R., \& Fleming, T. A. 1987, BAAS, 19, 643

Hajduk, M., Zijlstra, A., \& Gesicki, K. 2010, MNRAS, 406, 626

Hamuy, M., Walker, A. R., Suntzeff, N. B., et al. 1992, PASP, 104, 533

Handler, G. 2003, IAUS, 209, 237

Harrington, J. P., \& Paltoglou, G. 1993, ApJ, 411, L103

Heber, U., \& Drilling, J. S. 1984, MitAG, 62, 252

Hewett, P., \& Irwin, M. 2004, INGN, 8, 6

Hillwig, T. C., Bond, H. E., \& Afsar, M. 2006, IAUS, 234, 421

Hsia, C. H., Ip, W. H., \& Li, J. Z. 2006, AJ, 131, 3040

Hultzsch, P. J. N., Puls, J., Méndez, R. H., et al. 2007, A\&A, 467, 1253

Hyung, S., Aller, L. H., \& Feibelman, W. A. 1999, ApJ, 525, 294

Iben, I., Jr., Kaler, J. B., Truran, J. W., \& Renzini, A. 1983, ApJ, 264, 605

Jones, D. H. P., Evans, D. S., \& Catchpole, R. M. 1969, Obs., 89, 18

Kerber, F., Lercher, G., Sauer, W., Seeberger, R., \& Weinberger, R. 1994, AGAb, 10,172

Kerber, F., Mignani, R. P., Guglielmetti, F., \& Wicenec, A. 2003, A\&A, 408, 1029

Kingsburgh, R. L., \& Barlow, M. J. 1994, MNRAS, 271, 257

Kondrateva, L. N. 1994, AstL, 20, 644

Kraus, M., Borges Fernandes, M., de Araújo, F. X., \& Lamers, H. J. G. L. M. 2005, A\&A, 441, 289

Kudritzki, R. P., Simon, K. P., \& Méndez, R. H. 1981, Msngr, 26, 7

Kwitter, K. B., Congdon, C. W., Pasachoff, J. M., \& Massey, P. 1989, AJ, 97, 1423

Lamers, H. J. G., Zickgraf, F., de Winter, D., Houziaux, L., \& Zorec, J. 1998, A\&A, 340, 117

Law, W. Y., \& Ritter, H. 1983, A\&A, 123, 33

Lawlor, T. M., \& MacDonald, J. 2001, ASPC, 226, 20

Lee, T.-H., Stanghellini, L., Ferrario, L., \& Wickramasinghe, D. 2007, AJ, 133, 987

Liu, X. W., Storey, P. J., Barlow, M. J., et al. 2000, MNRAS, 312, 585

Lutz, J. H. 1977, A\&A, 60, 93

Lutz, J. H., \& Kaler, J. B. 1987, BAAS, 19, 1090

Mampaso, A., Corradi, R. L. M., Viironen, K., et al. 2006, A\&A, 458, 203

Margon, B., Downes, R. A., \& Katz, J. I. 1981, Nature, 293, 200

Méndez, R. H. 1991, IAUS, 145, 375

Méndez, R. H., \& Niemela, V. S. 1977, MNRAS, 178, 409

Méndez, R. H., \& Niemela, V. S. 1981, ApJ, 250, 240

Méndez, R. H., \& Niemela, V. S. 1982, IAUS, 99, 457

Méndez, R. H., Kudritzki, R. P., Herrero, A., Husfeld, D., \& Groth, H. G. 1988a, A\&A, 190, 113

Méndez, R. H., Kudritzki, R. P., Groth, H. G., Husfeld, D., \& Herrero, A. 1988b, A\&A, 197, L25

Miranda, L. F., Vazquez, R., Torrelles, J. M., Eiroa, C., \& Lopez, J. A. 1997, MNRAS, 288, 777

Miszalski, B. 2010, Asymmetric Planetary Nebulae V

Miszalski, B., Parker, Q. A., Acker, A., et al. 2008, MNRAS, 384, 525

Miszalski, B., Acker, A., Moffat, A. F. J., Parker, Q. A., \& Udalski, A. 2009a, A\&A, 496, 813

Miszalski, B., Acker, A., Parker, Q. A., \& Moffat, A. F. J. 2009b, A\&A, 505, 249

Miszalski, B., Corradi, R. L. M., Jones, D., et al. 2010, in press

Mitchell, D. L., O’Brien, T. J., Pollacco, D., \& Bryce, M. 2007a, IAUS, 240, 429

Mitchell, D. L., Pollacco, D., O'Brien, T. J., et al. 2007b, MNRAS, 374, 1404

Morgan, D. H., Parker, Q. A., \& Russeil, D. 2001, MNRAS, 322, 877 
Napiwotzki, R. 1999, A\&A, 350, 101

Napiwotzki, R., Tovmassian, G., Richer, M. G., et al. 2005, AIPC, 804, 173 Parker, Q. A., \& Morgan, D. H. 2003, MNRAS, 341, 961

Parker, Q. A., Acker, A., Frew, D. J., et al. 2006, MNRAS, 373, 79

Peña, M., \& Medina, S. 2002, RMxAA, 38, 23

Peña, M., Torres-Peimbert, S., \& Ruiz, M. T. 1992, A\&A, 265, 757

Peña, M., Peimbert, M., Torres-Peimbert, S., Ruiz, M. T., \& Maza, J. 1995, ApJ, 441,343

Peña, M., Ruiz, M. T., Bergeron, P., Torres-Peimbert, S., \& Heathcote, S. 1997, A\&A, 317, 911

Pereira, C. B. 2004, A\&A, 413, 1009

Pereira, C. B., Miranda, L. F., Smith, V. V., \& Cunha, K. 2008, A\&A, 477, 535

Pierce, M. J., Frew, D. J., Parker, Q. A., \& Köppen, J. 2004, PASA, 21, 334

Pottasch, S. R. 1983, ASSL, 107

Pottasch, S. R. 1996, A\&A, 307561

Rauch, T., Köppen, J., Napiwotzki, R., \& Werner, K. 1999, A\&A, 347, 169

Rauch, T., Heber, U., \& Werner, K. 2002, A\&A, 381, 1007

Rodríguez, M., Corradi, R. L. M., \& Mampaso, A. 2001, A\&A, 377, 1042

Sabbadin, F., Falomo, R., \& Ortolani, S. 1987, A\&AS, 67, 541
Santander-García, M. 2010, Asymmetric Planetary Nebulae V Saurer, W., Werner, K., \& Weinberger, R. 1997, A\&A, 328, 598 Seaton, M. J. 1979, MNRAS, 187, 785

Shen, Z. X., Liu, X. W., \& Danziger, I. J. 2004, A\&A, 422, 563

Smith, N., Bally, J., \& Walawender, J. 2007, AJ, 134, 846

Soker, N., \& Zucker, D. B. 1997, MNRAS, 289, 665

Stanghellini, L., Kaler, J. B., \& Shaw, R. A. 1994, A\&A, 291, 604

Tamura, S., \& Shaw, R. A. 1987, PASP, 99, 1264

Tovmassian, G. H., Napiwotzki, R., Richer, M. G., et al. 2004, ApJ, 616, 485

Tweedy, R. W., \& Kwitter, K. B. 1996, ApJS, 107, 255

Tylenda, R., \& Górny, S. K. 1993, AcA, 43, 389

Tylenda, R., Acker, A., \& Stenholm, B. 1993, A\&AS, 102, 595

Walsh, J. R., \& Walton, N. A. 1996, A\&A, 315, 253

Weidmann, W. A., Gamen, R., Díaz, R. J., \& Niemela, V. S. 2008, A\&A, 488, 245

Weinberger, R., Kerber, F., \& Groebner, H. 1997, A\&A, 323, 963

Werner, K., \& Herwig, F. 2006, PASP, 118, 183

Wlodarczyk, K., \& Olszewski, P. 1994, AcA, 44, 407

Zhang, C. Y., \& Kwok, S. 1991, A\&A, 250, 179

Zijlstra, A., Pottasch, S., \& Bignell, C. 1990, A\&AS, 82, 273 


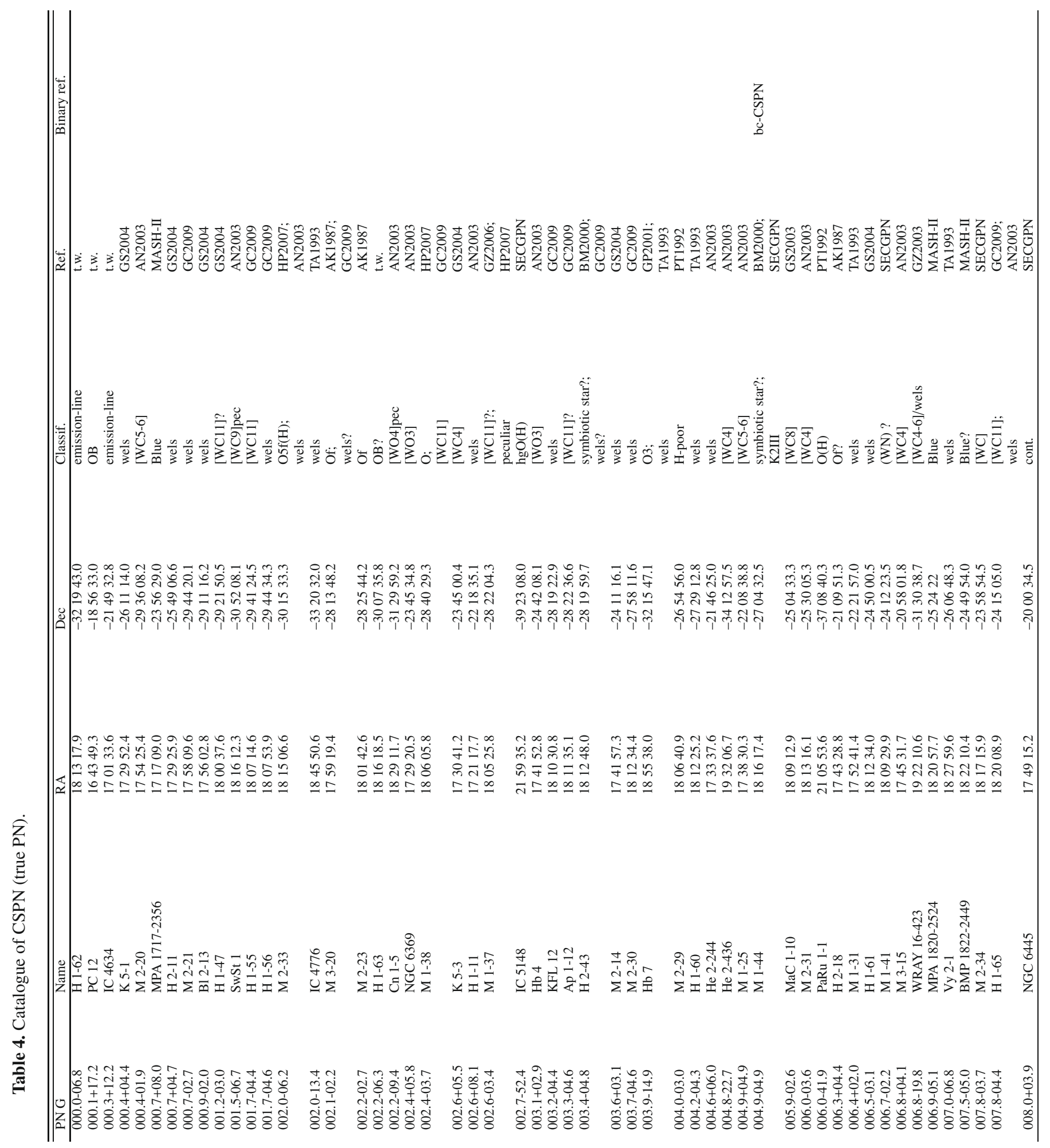




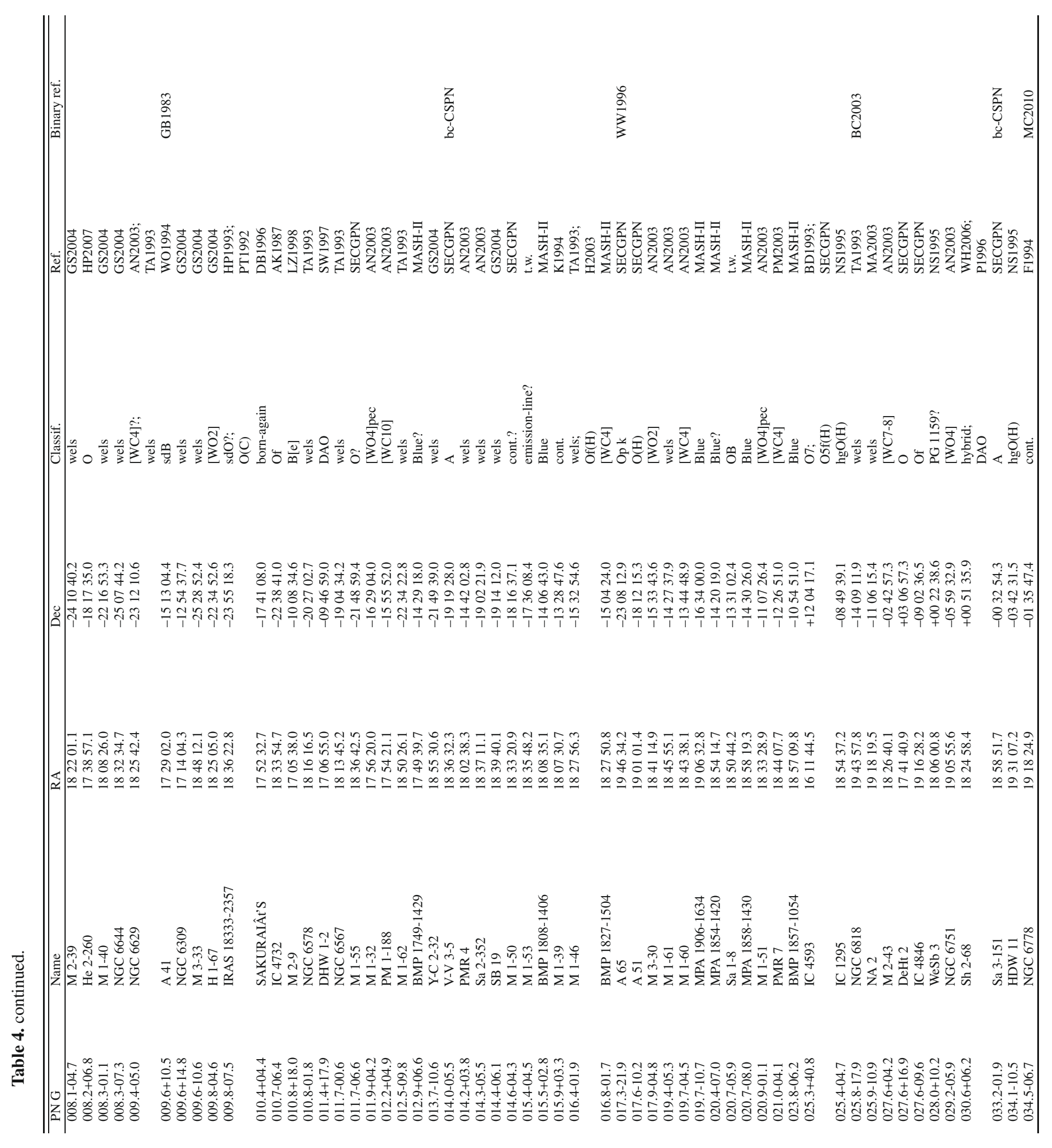


W. A. Weidmann and R. Gamen: Central stars of planetary nebulae: New spectral classifications and catalogue

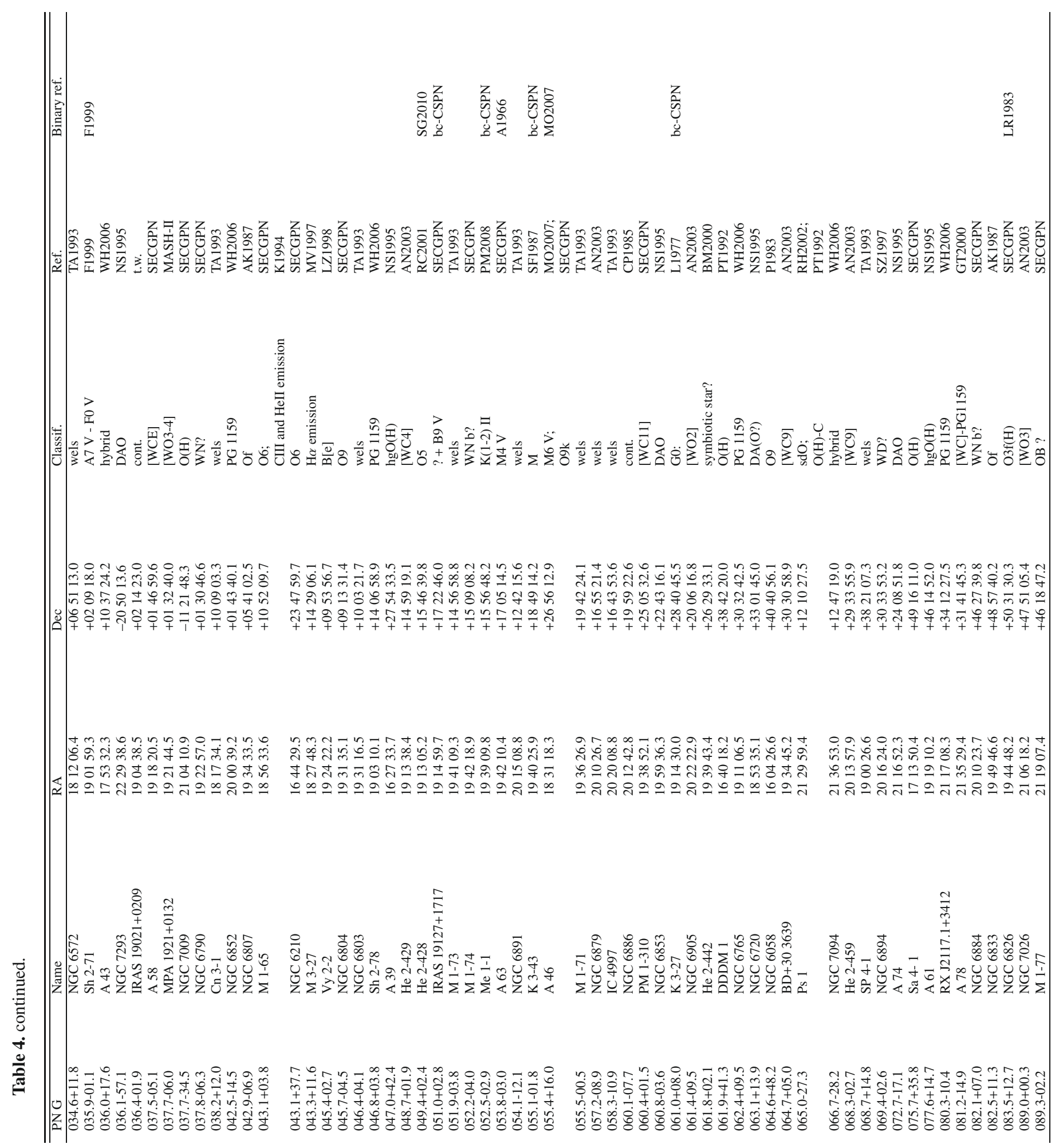




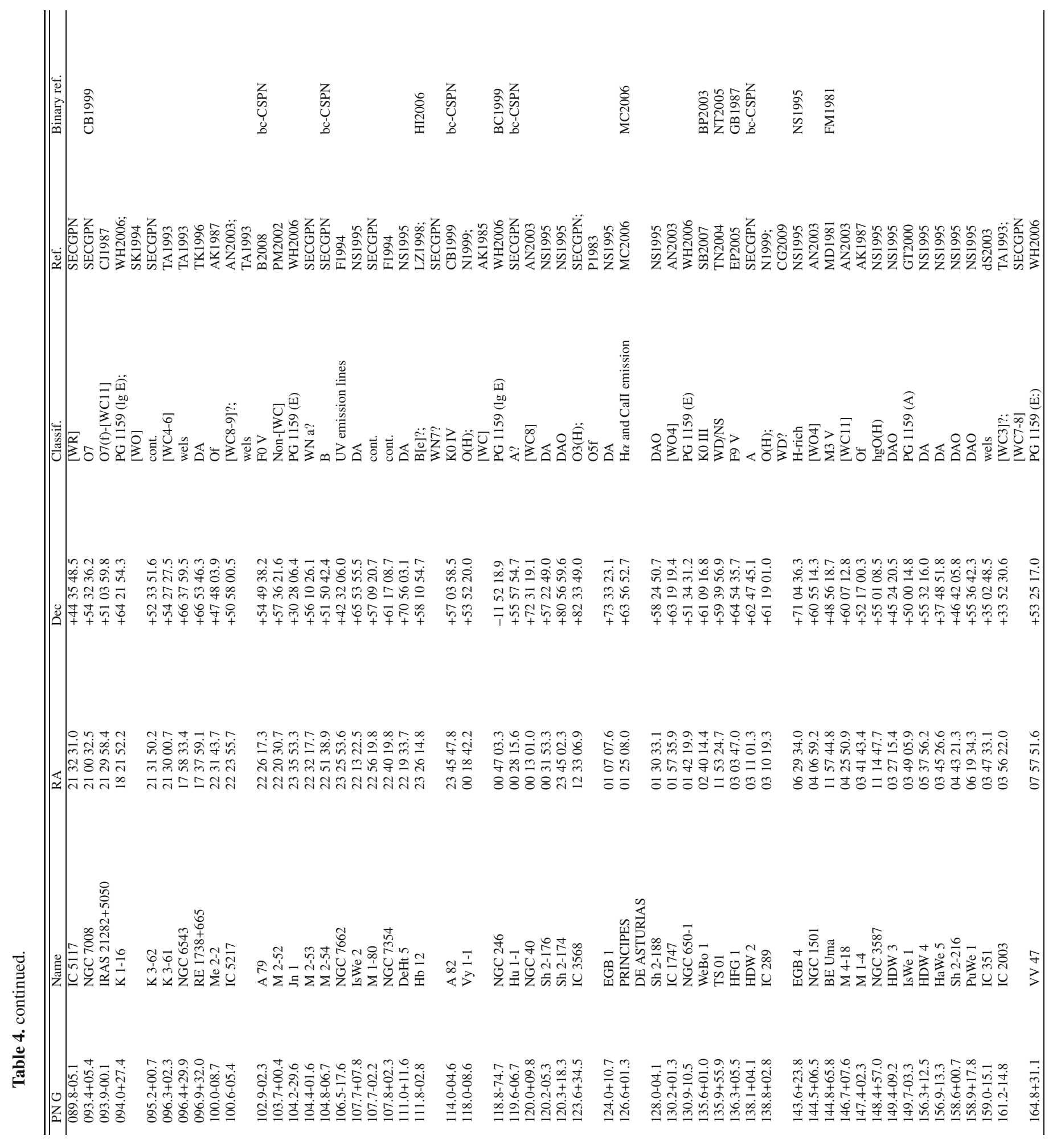


W. A. Weidmann and R. Gamen: Central stars of planetary nebulae: New spectral classifications and catalogue

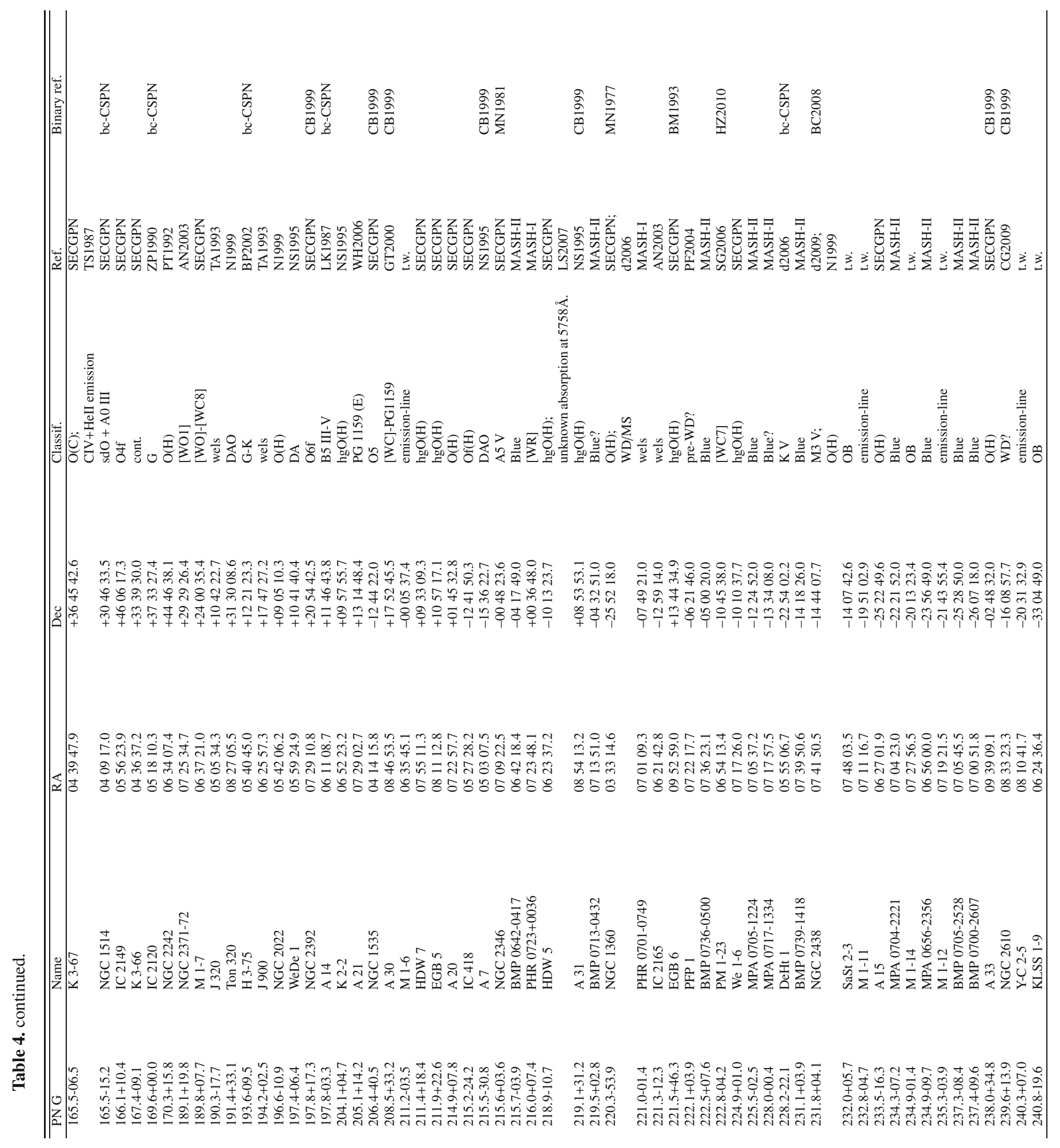




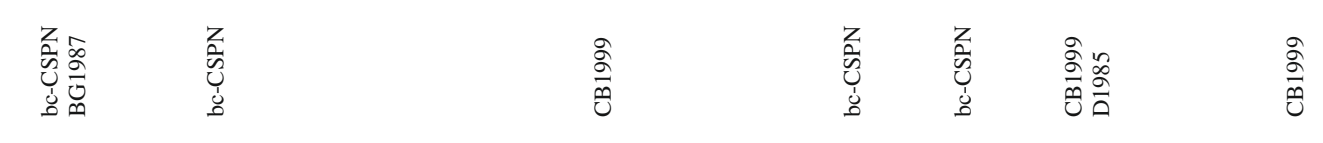

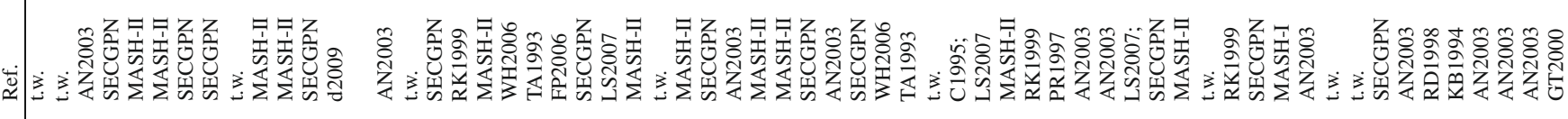

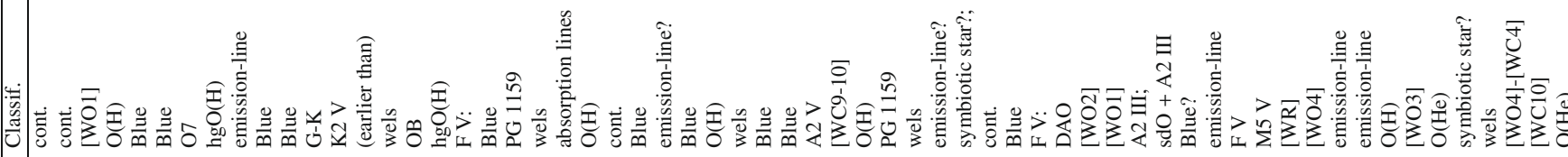

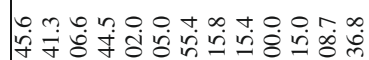

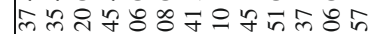

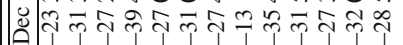

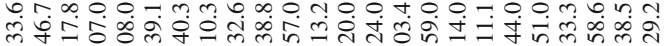

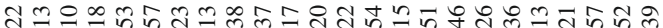

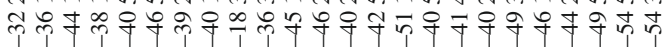

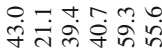
ले 8 in

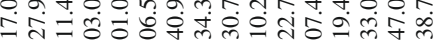
ยู่

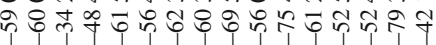

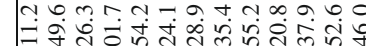

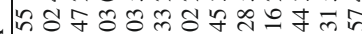

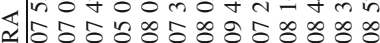

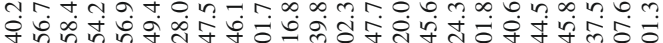

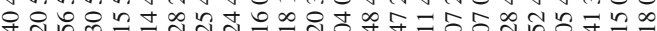

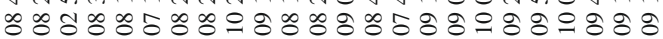

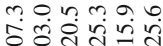

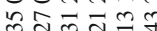
$\therefore 8$ \& 80

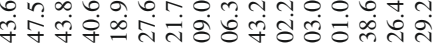

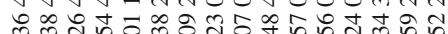
\& $8=0$ 응ㅇㅇㅇㅇㅇㅇㅇㅢ

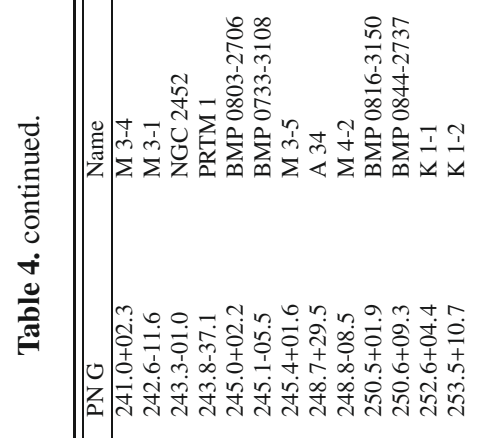

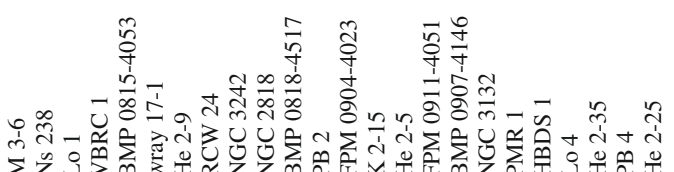

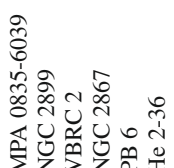

ڤิ

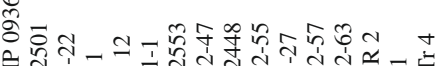

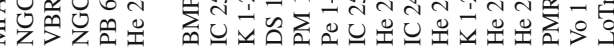

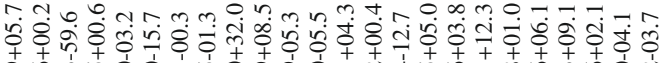

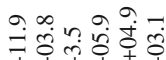

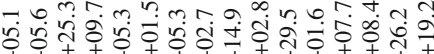
m.jun

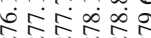

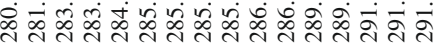


W. A. Weidmann and R. Gamen: Central stars of planetary nebulae: New spectral classifications and catalogue

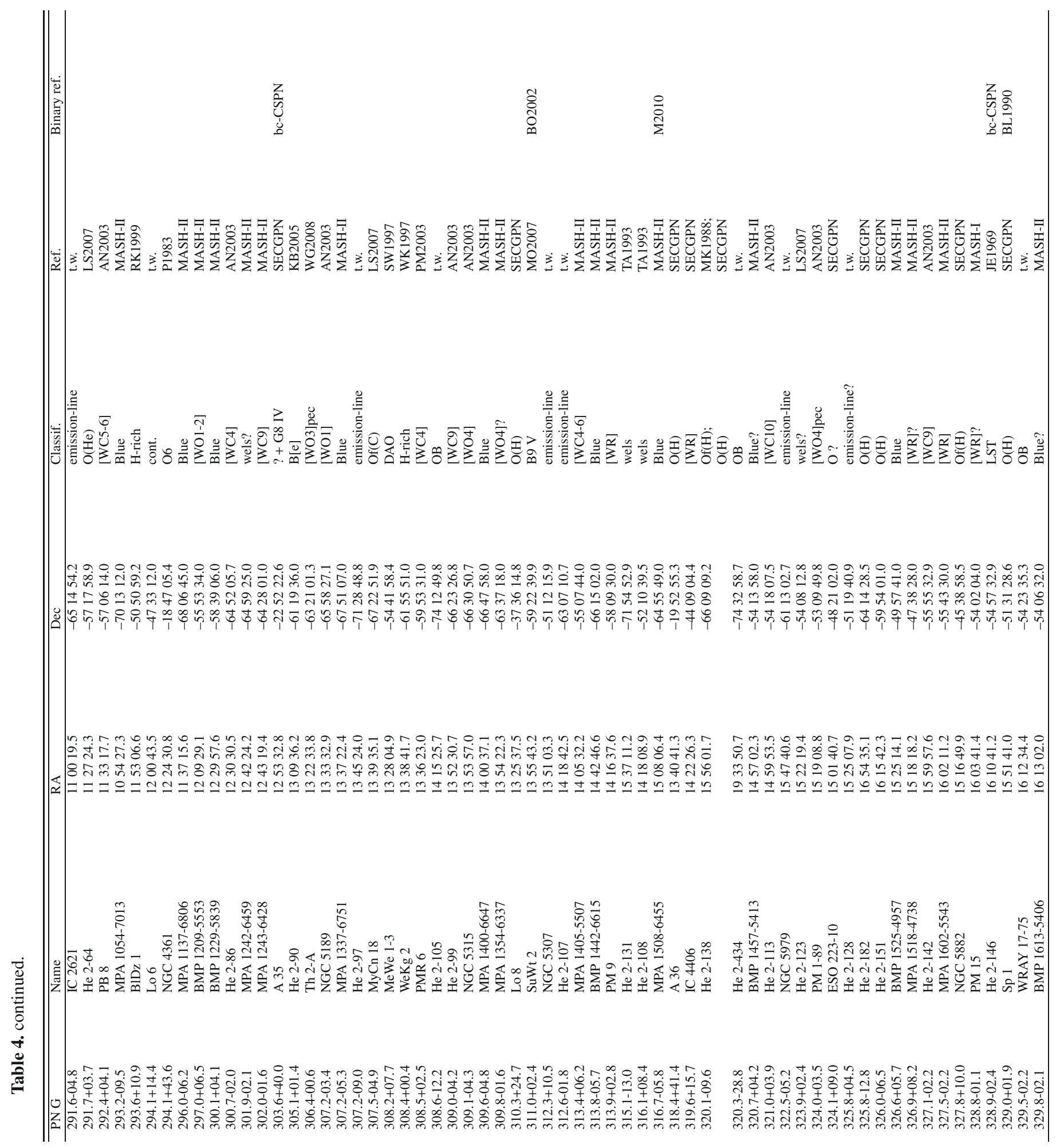




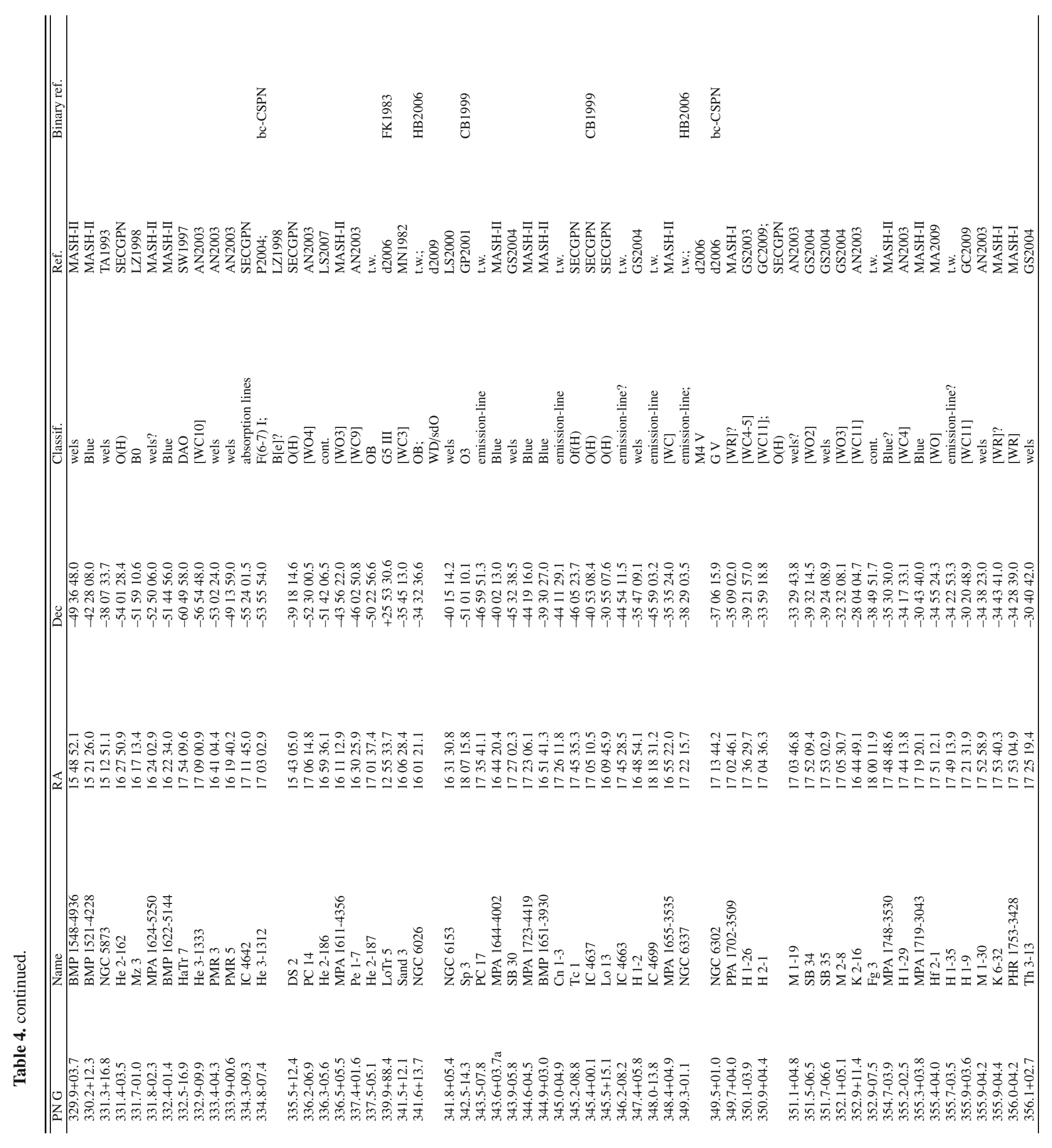



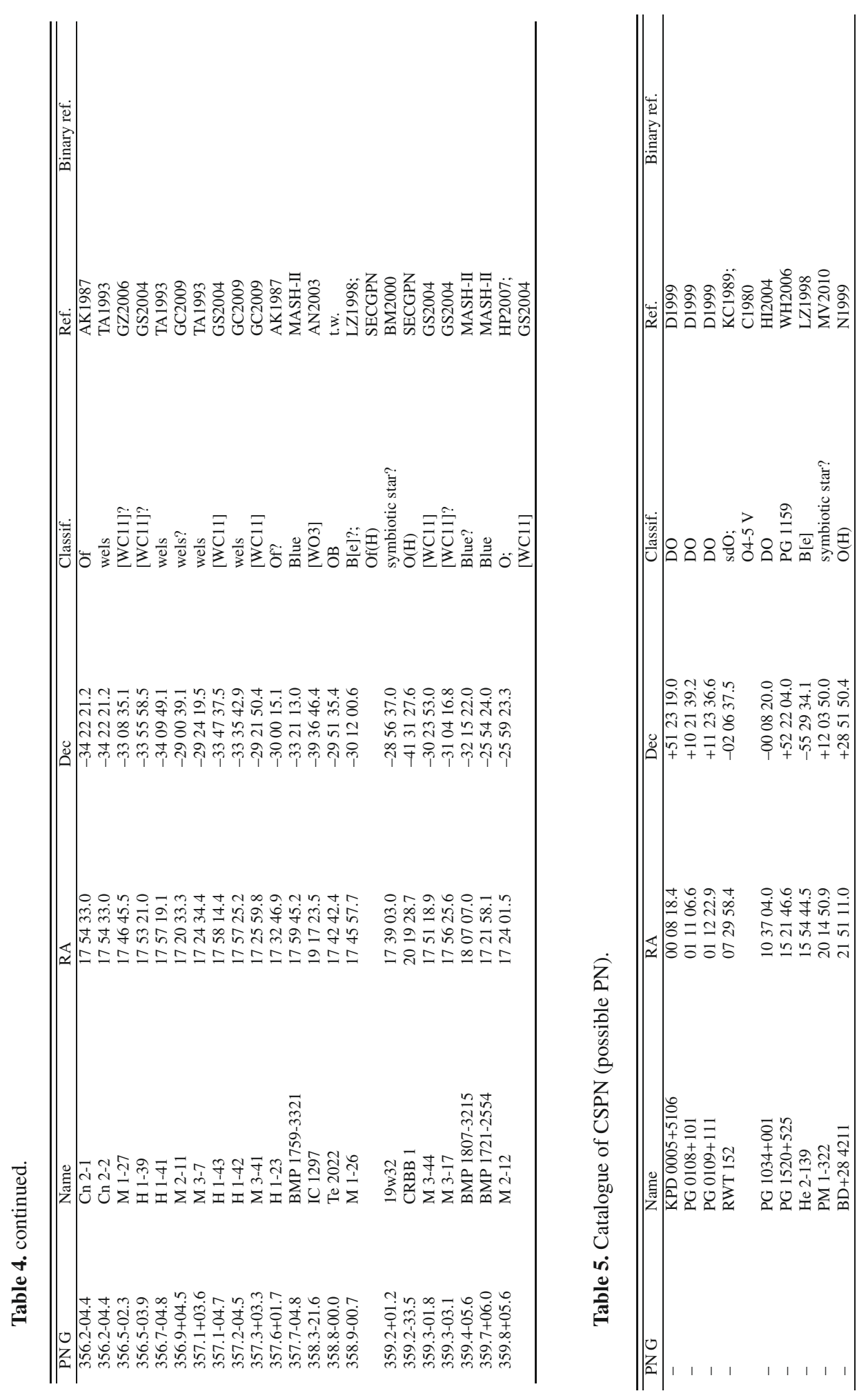
References to Tables 4 and 5:

A1966 - Abell (1966);

AK1985 - Aller \& Keyes (1985);

AK1987 - Aller \& Keyes (1987);

AN2003 - Acker \& Neiner (2003);

CB2008 - Bilikova et al. (2008);

BC1999 - Bond \& Ciardullo (1999);

BC2003 - Benetti et al. (2003);

B2008 - Bohigas (2008);

BD1993 - Bianchi \& Defrancesco (1993);

BG1987 - Bond \& Grauer (1987);

BL1990 - Bond \& Livio (1990);

BM1993 - Bond et al. (1993);

BM2000 - Belczyski ă et al. (2000);

BO2002 - Bond et al. (2002);

BP2002 - Bond \& Pollacco (2002);

BP2003 - Bond et al. (2003);

CB1999 - Ciardullo et al. (1999);

C1980 - Chromey (1980);

C1995 - Corradi (1995);

CB1999 - Ciardullo et al. (1999);

CG2009 - Chu et al. (2009);

CJ1987 - Cohen \& Jones (1987);

CP1985 - Cerruti-Sola \& Perinotto (1985);

D1983 - Drilling (1983);

D1985 - Drilling (1985);

D1999 - Dreizler (1999);

d2006 - de Marco (2006);

d2009 - de Marco (2009);

DB1996 - Duerbeck \& Benetti (1996);

dS2003 - de Marco et al. (2003);

EP2005 - Exter et al. (2005);

F1999 - Feibelman (1999);

F1994 - Feibelman (1994);

FK1983 - Feibelman \& Kaler (1983);

FM1981 - Ferguson et al. (1981);

FP2006 - Frew et al. (2006);

FS2010 - Frew et al. (2010);

GB1983 - Grauer \& Bond (1983);

GB1987 - Grauer et al. (1987);

GC2009 - Górny et al. (2009);

GP2001 - Gauba et al. (2001);

GS2003 - Górny \& Sidmiak (2003);

GS2004 - Górny et al. (2004);

GT2000 - Górny \& Tylenda (2000);

GZ2003 - Gesicki \& Zijlstra (2003);

GZ2006 - Gesicki et al. (2006);

HB2006 - Hillwig (2006);

HI2006 - Hsia et al. (2006);

HZ2010 - Hajduk et al. (2010);

H2003 - Handler (2003);

HD1984 - Heber \& Drilling (1984);

HI2004 - Hewett \& Irwin (2004);

HP1993 - Harrington \& Paltoglou (1993);

HP2007 - Hultzsch et al. (2007);

JE1969 - Jones et al. (1969);

K1994 - Kondrat'eva (1994);

KB1994 - Kingsburgh \& Barlow (1994);
KB2005 - Kraus et al. (2005);

KC1989 - Kwitter et al. (1989);

L1977 - Lutz (1977);

LK1987 - Lutz \& Kaler (1987);

LS2000 - Liu et al. (2000);

LS2007 - Lee et al. (2007);

LR1983 - Law \& Ritter (2001)

LZ1998 - Lamers et al. (1998);

MA2003 - Marcolino \& derajoă (2003);

MA2009 - Miszalski et al. (2009a);

MA2010 - Miszalski (2010);

MASH-I - Parker et al. (2006);

MASH-II - Miszalski et al. (2008);

MC2006 - Mampaso et al. (2006);

MC2010 - Miszalski et al. (2010);

MD1981 - Margon et al. (1981);

MK1988 - Méndez et al. (1988a);

MN1977 - Méndez \& Niemela (1977);

MN1982 - Méndez \& Niemela (1982);

MN1981 - Méndez \& Niemela (1981);

MO2007 - Mitchell et al. (2007a);

MP2001 - Morgan et al. (2001);

MP2007 - Mitchell et al. (2007b);

MV1997 - Miranda et al. (1997);

MV2010 - Miranda et al. (2010);

N1999 - Napiwotzki (1999);

NS1995 - Napiwotzki \& Schoenberner (1995);

NT2005 - Napiwotzki et al. (2005);

P1983 - Pottasch (1983);

P1996 - Pottasch (1996);

P2004 - Pereira (2004);

PF2004 - Pierce et al. (2004);

PM2002 - Pea \& Medina (2002);

PM2003 - Parker \& Morgan (2003);

PM2008 - Pereira et al. (2008);

PR1997 - Pena et al. (1997);

PT1992 - Pena et al. (1992);

RC2001 - Rodrnguez et al. (2001);

RD1998 - Rauch et al. (1998);

RH2002 - Rauch et al. (2002);

RK1999 - Rauch et al. (1999);

SB2007 - Smith et al. (2007);

SECGPN - Acker et al. (1992);

SF1987 - Sabbadin et al. (1987);

SG2010 - Santander-García (2010);

SK1994 - Stanghellini et al. (1994);

SL2004 - Shen et al. (2004);

SW1997 - Saurer et al. (1997);

SZ1997 - Soker \& Zucker (1997);

TA1993 - Tylenda et al. (1993);

TK1996 - Tweedy \& Kwitter et al. (1996);

TN2004 - Tovmassian et al. (2004);

TS1987 - Tamura \& Shaw (1987);

WG2008 - Weidmann et al. (2008);

WH2006 - Werner \& Herwig (2006);

WK1997 - Weinberger et al. (1997);

WW1996 - Walsh \& Walton (1996);

WO1994 - Wlodarczyk \& Olszewski (1994);

ZP1990 - Zijlstra et al. (1990). 